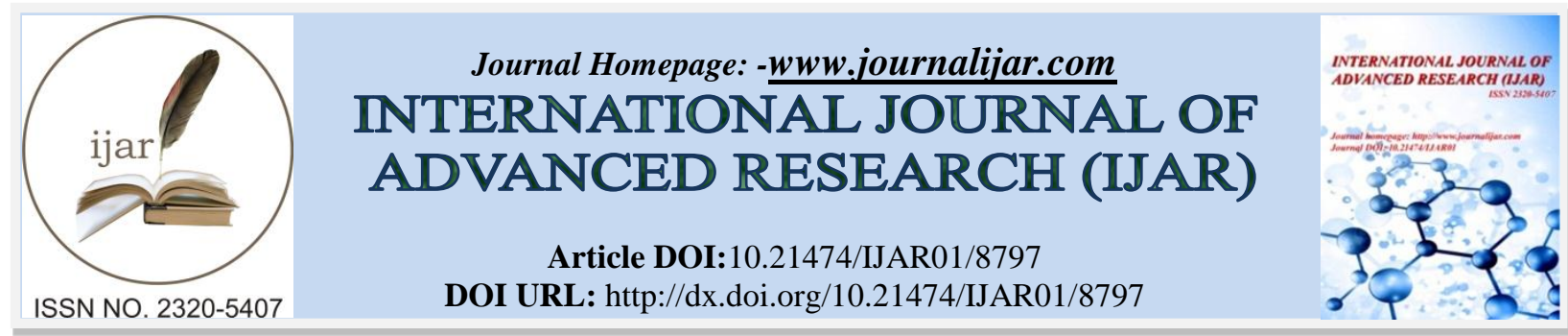

RESEARCH ARTICLE

\title{
CLINICAL ATTACHMENT LEVEL: AN UNSUNG HERO IN PERIODONTAL DIAGNOSIS.
}

\section{Dr. Shaswata Karmakar and Dr. Shobha Prakash.}

1. Senior Lecturer, Department of Periodontics, Awadh Dental College and Hospital Jamsedpur.

2. Professor and Head, Department of Periodontics, College of Dental Sciences Davangere.

\section{Manuscript Info}

Manuscript History

Received: 03 February 2019

Final Accepted: 05 March 2019

Published: April 2019

Key words:-

Clinical attachment level, Relative attachment level, Periodontal diagnosis, Probing pocket depth.

\section{Abstract}

The initial lesion in the development of periodontitis is the inflammation of the gingival tissue in response to bacterial challenge. Attachment loss begins as an inflammatory change in the connective tissue wall of the gingival sulcus when the tissue loses its cohesiveness and detaches from the tooth surface. In current clinical practice, primarily used objective parameters to measure the tissue destruction are Probing pocket depth (PD), Clinical attachment level (CAL) and Relative attachment level (RAL). The assessment of CAL provides information relating to the loss or gain of connective tissue attachment to the root surface, and represent the standard by which clinicians record disease status. Though histological examination is the gold standard and periodontal probing is the most widely accepted method for measuring the amount of attachment loss, CAL remains the unsung hero in periodontal diagnosis.

Copy Right, IJAR, 2019,. All rights reserved.

\section{Introduction:-}

Periodontitis is the inflammation of the periodontium. Increased probing depth and loss of clinical attachment are pathognomonic for periodontitis. The three general approaches that are used to clinically assess damage to the periodontal tissues are: 1) visual detection of clinical signs of tissue destruction, 2) measurement of clinical attachment level with a periodontal probe, and 3) radiographic detection of bone loss. In current clinical practice, primarily used objective parameters to measure the tissue destruction are:

1. Probing pocket depth (PD) - It is the distance from the gingival margin to the base of the probeable crevice.

2. Clinical attachment level (CAL) - It is the distance from the cementoenamel junction (CEJ) to the base of the probeable crevice.

3. Relative attachment level (RAL) - It is the distance from the fixed landmark other than CEJ to the base of the probeable crevice. When the CEJ is not detectable or is missing due to a dental restoration, CAL cannot be measured. In such situation, another fixed landmark such as margin of the restoration, the edge of a stent, or the occlusal surface of the tooth.

\section{Pathogenesis Of Attachment Loss - An Overview}

The initial lesion in the development of periodontitis is the inflammation of the gingival in response to bacterial challenge. Attachment loss begins as an inflammatory change in the connective tissue wall of the gingival sulcus. The cellular and fluid inflammatory exudates causes degeneration of the surrounding connective tissue, including the gingival fibers. Just apical to junctional epithelium (JE), collagen fibers are destroyed and the area becomes 
occupied by the inflammatory cells and edema. As a consequence of loss of collagen, the apical cells of JE proliferate along the root. The coronal portion of JE detaches from the root as the apical portion migrates. When the relative volume of PMNs reaches approx $60 \%$ or more of JE, the tissue loses its cohesiveness and detaches from the tooth surface, resulting in Loss of attachment ${ }^{1}$. In their classic paper Socransky et al (1984) ${ }^{2}$ provided a much needed landmark discussion on the nature of progression of periodontitis. They pointed out that the prevailing model of the disease had been of a slow, continuous process of attachment loss, and that much data had accumulated to suggest that progression might occur as bursts, interspersed with quiescent phases at individual sites. 2 types of bursts were suggested, one described as occurring randomly with time in an individual and the other where a number of sites lost attachment within a short period of time and then remained largely quiescent.

\section{Mesurement of attachment level - CAL and RAL:}

1. Periodontal probing

2. Comparison of pre-extraction measurements of AL with post-extraction estimates of probe penetration relative to connective tissue attachment level on root surfaces.

3. Direct or in situ histological assessment of probe penetration

4. Fibre optic micro-periodontoscopy - Periodontoscopy® System

\section{Periodontal probing:}

Periodontal probing is the insertion of the periodontal probe into the sulcus or the clinical pocket parallel to the long axis of the tooth and applying the force to move it apically into the tissue along the tooth surface. The probe is "walked" circumferentially around each surface of tooth to detect the areas of deepest penetration at each of six tooth surfaces: distofacial, facial, mesiofacial, distolingual, lingual and mesiolingual. The gingival margin position may be recorded as a positive number when the marginal gingiva is apical to CEJ (recession), or as a negative no. if the marginal gingiva is coronal to CEJ. Once the probing depth (PD) is determined, CAL is calculated as PD + gingival margin to CEJ measurement. Eg:if a site has $2 \mathrm{~mm}$ recession and PD of $5 \mathrm{~mm}$, then CAL is $7 \mathrm{~mm}(2+5)$. Conversely, if the gingival margin is located $2 \mathrm{~mm}$ coronal to CEJ, and $5 \mathrm{~mm}$ PD is present, the CAL is $3 \mathrm{~mm}(-2$ $+5)$. In some cases, the gingival margin is located at the CEJ, in which case the PD and CAL are same. As a general rule, a probe reading that falls between two calibrated marks on the probe should be rounded upward to the next highest millimeter ${ }^{3}$. Philstrom (1972) ${ }^{4}$ have classified periodontal probes into three generations i.e First generation (or Conventional) probes, Second generation probes or pressure-sensitive probes and Third generation probes or pressure controlled electronic probes with direct computer data capture. Watts et al $(1995)^{5}$ have made an analysis of the problems associated with unidimensional probing, namely its validity and reliability, and has lead to an approach to accurate clinical measurement of periodontitis in 3-dimensions. They stated that an approach of probing around until the maximum depth of a pocket is reached is invalid for detection of a change in attachment level, in the event of horizontal progression of disease widening the pocket and they have put forward the idea of a $4^{\text {th }}$ and $5^{\text {th }}$ generation instrument. $4^{\text {th }}$ generation probes are 3 dimensional probes in which sequential probe positions are made and $5^{\text {th }}$ generation probes or ultrasonographic probes. Listgarten $(1980)^{6}$ stated that probing depth measured from gingival margin seldom correspond to histological sulcus or pocket depth. The discrepancy was less in the absence in the inflammatory changes and increased with increasing degrees of inflammation. Following treatment, decreased probing depth measurements may be due in part to decreased penetrability of the gingival tissues. The results of the study by Van Der Velden (1979) ${ }^{7}$ indicated that

1. Using the probe of $0.63 \mathrm{~mm}$ diameter, the optimal force level for clinical periodontal measurements is $0.75 \mathrm{~N}$.

2. A force of $0.75 \mathrm{~N}$ is well tolerated and meets a few objections from the patients.

3. When a probing force of $0.75 \mathrm{~N}$ is used, the tip of the probe in both shallow and deep periodontal pockets is located at the most coronal intact connective tissue fibers.

4. A plateau value in pocket depth measurements is found when a probing force of $1.25 \mathrm{~N}$ is employed.

5. The mean width of the connective tissue attachment is approximately $1.9 \mathrm{~mm}$.

Abbas et al (1982) $)^{8}$ in an investigation studied the effect of standardization of probing spot and direction of probe insertion into the pocket in conjunction with a probing force of $0.75 \mathrm{~N}$ on the reproducibility of pocket depth measurements. The results indicated that only a training program together with a standardized probing force leads to reproducible interproximal pocket depth measurements.

Assessment Of Clinical Attachment Level At Furcation:

Since the furcation has a vertical and horizontal component, conventional straight probes may also underestimate the severity of the furcation involvement. Vertical attachment level (CAL-V) and Horizontal attachment level (CAL-H) 
at the furcation area may be measured. The respective landmark from where the horizontal loss of attachment is frequently estimated is an imaginary tangent applied at both roots or root cones at the level of the furcation entrance. If the furcation entrance is located subgingivally, the respective concavity of the root surface may be probed first (Miller 1999) ${ }^{9}$. When ignoring the horizontal component of the pocket, vertical measurements in the facial furcation area employing a pressure controlled probe set at $0.5 \mathrm{~N}$ yielded a penetration of the probe of about 2 $\mathrm{mm}$ into the inflamed connective tissue, on average, whereas the probe tip was located a mean $0.4 \mathrm{~mm}$ apical to the alveolar crest (Moriarty et al. 1988) ${ }^{10}$. A plausible explanation may be the difficulty to follow the contour of the tooth with the probe in the furcation area as compared to the flat surfaces of single-rooted teeth. Unfortunately, the design of the constant force probe generally prevented horizontal probing of most of the facial and lingual furcations was not possible because of anatomical characteristics as gingival height and a shallow vestibular sulcus. Clearly, the pressure-controlled, straight probe seems not to be suitable for measuring horizontal attachment loss in furcations. The reproducibility of horizontal measurements was somewhat better at sites with a vertical dimension of the furcation in the $>1$ to $2 \mathrm{~mm}$ range (0.78) as compared to shallower or higher furcations $(0.57$ and 0.64 , respectively). Eickholz (1995) ${ }^{11}$ estimated the validity and reliability of measurements with regard to the horizontal attachment loss in furcations in considerable detail. These authors investigated Nabers' curved, colour-coded probe with $3 \mathrm{~mm}$ increments and subsequently compared the results with a rigid and a flexible, pressure-controlled probe. The error of furcation measurement also seemed to depend on the depth of the lesion, paradoxically with a lower reproducibility at sites with no or incipient involvement. Moriarty et al (1988) ${ }^{10 .}$ Eickholz et al (1998) ${ }^{11}$ did a study to investigate the reliability of the assessment of clinical furcation parameters (horizontal attachment levels (CAL$\mathrm{H}$ ), class of furcation invasion) using a Naber's, TPS and a PCP-UNC 15-probe. They concluded that clinical diagnosis of furcation lesions using the $3 \mathrm{~mm}$ incrementally marked Naber's probe provides reproducible and valid information about furcation invasion. Eickholz et al (1994) ${ }^{12}$ again studied the furcation involvement of 100 molars in 25 patients suffering from moderate to advanced periodontitis. Presurgically, the horizontal probing attachment level (PAL-H) within the furcations was assessed and the measurements were repeated intrasurgically and compared with presurgical assessments of furcation degrees. There was no significant difference between furcation degree as assessed presurgically and intrasurgically.

\section{Significance of CAL:}

Introduced by Ramjford, CAL is considered as a standard method for judging clinical response in the treatment of periodontitis. The assessment of CAL provides information relating to the loss or gain of connective tissue attachment to the root surface, and represent the standard by which clinicians record disease status. The severity of attachment loss is generally, but not always, correlated with the pocket depth. This is because the CAL depends on the location of the base of the pocket from the CEJ, whereas PD is the distance from the gingival margin to the base of the pocket; and the position of gingival margin is variable. Thus teeth with same pocket depth may be associated with different degrees of attachment loss, and pockets of different depths may be associated with same amount of attachment loss. CAL is the clinical approximation of the loss of connective tissue attachment from the root surface. They are extremely useful in clinically monitoring attachment level changes on a site-by-site basis from one visit to the next. In current clinical practice, CAL measurements are the primary basis upon which a clinician can be reasonably certain that a program of supportive periodontal therapy is working or not.

\section{Validity and reliability of periodontal probing:}

The validity of attachment level measurements describes the potential to detect the apical termination of the junctional epithelium, or in other words, the most coronal fibers of the attachment apparatus. Reliability of a diagnostic measure is its potential to reproduce the measurement after a short period to time in a reasonable and satisfactory way. Many studies clearly show that measurements of PD and CAL taken after a short time interval are reproducible to within $\pm 1 \mathrm{~mm}$ over $90 \%$ of the time. Reddy et al (1997) ${ }^{13}$ compared the intra-examiner and interexaminer error of 2 constant force probes to the reading of a conventional manual probe. Their data indicate that both manual and controlled-force probes can provide measurement within less than I mm of error. Grossi et al $(1996)^{14}$ in their study determined the relative contribution to probing measurement reliability of several factors, compared with that of random error. Probing measurements were performed by examiners properly trained and calibrated. Individual examiner, individual patient and site location contribute up to $10 \%$ to the overall variance. Hence, the pattern of variability for intra-examiner variance of probing measurements performed with either electronic or conventional probes by trained and calibrated examiners is mostly random error. Breen et al (1997) ${ }^{15}$ compared relative attachment levels (RAL) and probeable crevice depths (PCD) from 6 periodontal probes. Significant inter-probe differences were found in RAL and PCD which have clinical importance in data interpretation and comparison. 


\section{Limitations of attachment level measurements:}

1. Although attachment level is used as an indicator of the amount of periodontal support at a specific location on the tooth, this measurement does not provide an accurate assessment of support in terms of 3-dimensions or root surface area.

2. CAL represents the evidence of past periodontal disease, and does not provide real markers of future or ongoing attachment loss.

3. The apical extent of periodontal probe penetration and depth measurement has been shown in many studies to be dependent on degree of inflammation, probing force, probe tip design (shape, thickness and diameter), angulation and position of probing, and root anatomy, particularly in furcation areas; and training of the operator.

4. Other factors that are likely to influence clinical measurement of attachment level include intra- and interexaminer reliability, training of the operator, patient discomfort, accuracy of probe markings, and anatomical variations in tooth contours or position.

5. Measurement error may not be homogenous among tooth surfaces, probing depths or patients.

6. Errors in transcription which may occur while manually recording data may also result in accuracies.

\section{Risk Indicators For Future Attachment Loss:}

Grbic et al $(1991,1992)^{16,17}$, Grossi et al $(1994)^{18}$ and Haffajee et al $(1992)^{19}$ have studied various factors which can cause $\mathrm{AL}$ at a monitored site during the 6 months of monitoring.

1. Age -With increasing age the \% of subjects showing severe mean AL increased.

2. Systemic diseases -Diabetes and angina appear as the only systemic diseases correlated with AL. Allergy and anemia were negatively correlated with AL.

3. Smoking - There is a trend for increasing severity of AL with increasing packyears.

4. Microbial flora - Grossi et al (1994) found B. forsythus and P. gingivalis are the only two microorganisms showing increasing percentage of positive subjects from healthy to severe AL group.

5. Occupational hazards - The occupational hazards assessed included chemicals, asbestos, radiation. They found that mean AL was significantly greater for the individuals exposed to chemicals and asbestos compared to non-exposed.

6. Gender - Machtei et al (1992) ${ }^{20}$ found that males exhibited higher mean CAL values than females for all measured parameters.

7. Baseline mean attachment level - The presence of existing periodontal disease is a significant risk factor for future AL. The clinical entity of "established periodontitis" is suggested based on the presence of CAL $\geq 6 \mathrm{~mm}$ in two or more teeth \& one or more sites with PPD $\geq 5 \mathrm{~mm}^{23}$.

8. Baseline mean probing depth - increased probing depth increases the risk for future $\mathrm{AL}$

9. Baseline mean recession - increased recession increases the risk for future $\mathrm{AL}$

10. Baseline bleeding on probing - an increased incidence of BOP at baseline is associated with AL.

11. Number of missing teeth - As the no. of teeth decreased, the risk for future AL increased.

12. Tooth variable - Mandibular and maxillary molars and maxillary premolars were the teeth that displayed the highest incidence of clinical AL. maxillary anterior teeth and mandibular premolars demonstrated the lowest incidence of clinical AL.

13. Site variable - Sites with baseline probing depth of $\geq 8 \mathrm{~mm}$ demonstrated a significantly greater $\mathrm{AL}$ rate compared to sites with 4-7 mm existing pocket depth, which in turn had a significantly greater AL compared to sites with baseline probing depth of $\leq 3 \mathrm{~mm}$.

14. Subgingival temperature - Subjects with high mean subgingival temperature and widespread periodontal destruction appeared to be at greatest risk for new AL. Unchanging sites exhibited lower mean subgingival temp.

\section{Effect Of Periodontal Therapy / No Therapy On CAL}

The meta-analysis results (Hung et al 2002) ${ }^{21}$ show that periodontal probing depth (PD) and gain of attachment level do not improve significantly following root planing and scaling for patients with shallow initial periodontal probing depths. However, there was about a $1 \mathrm{~mm}$ reduction for medium initial periodontal probing depths and a 2 $\mathrm{mm}$ reduction for deep initial periodontal probing depths. There was about a $0.50 \mathrm{~mm}$ gain in attachment for medium initial periodontal probing depth measurements and slightly more than $1 \mathrm{~mm}$ gain in attachment for deep initial periodontal probing depth measurements. Surgical therapy for patients with deep initial periodontal probing depths showed better results than SRP in reducing pocket depths. When patients were followed up over 3 yrs or more, these differences were reduced to less than $0.4 \mathrm{~mm}$. Antibiotic therapy showed similar results to SRP. 
However, a consistent improvement in periodontal probing depth and gain of attachment is demonstrated when local antibiotic therapy is combined with SRP. Alves et al $(2005)^{22}$ did a study to clinically detect the immediate effect of root instrumentation with curettes and ultrasonic scalers on CAL. Within the limits of this study, it was concluded that root instrumentation causes a mean immediate attachment loss of $0.75 \mathrm{~mm}$, and that instrumentation with either curettes or ultrasonic scalers do not seem to reduce significantly the trauma resulting from instrumentation. Orobitg et al $(1996)^{23}$ investigated the relationship between pocket depth (PD) and attachment level (using Florida Probe) in a population that has not received dental treatment for at least $18 \mathrm{mths}$. Their study supports that change in PD is a poor indicator of attachment loss and disease progression. Therefore, repeated measurements of PD may not be used to substitute for repeated measurements of Relative attachment levels.

\section{Conclusion:-}

A variety of probing methods are available to measure CAL or RAL. All are currently dependent on a number of factors which have an impact on their use and interpretation. The selection of a particular probing method is dependent on its specific application. Probing depth changes time to time, depending on the position of gingival margin, which is unrelated to existing attachment on tooth. Whereas in case of CAL which is caused only with true loss of attachment. Though histological examination is the gold standard and periodontal probing is the most widely accepted method for measuring the amount of attachment loss, CAL remains the unsung hero in periodontal diagnosis.

\section{Acknowledgement:-}

Nil

\section{Funding:}

Nil

\section{Competing Interests:}

Nil

\section{Reference:-}

Carranza's Clinical Periodontology $-10^{\text {th }}$ ed

1. Socransky et al. New concepts of destructive periodontal disease. JCP 1984; 11: 21-32

2. Periodontal Medicine, Surgery and Implants - Rose, Genco

3. Philstrom BL. Measurement of attachment level in clinical trials: Probing methods. JP 1992; 63: 1072 - 1077

4. Watts et al. Periodontal disease activity: a development strategy for its investigation by means of accurate 3dimensional clinical measurement. JCP 1995; 22: 201-207

5. Listgarten Periodontal probing: What does it mean? JCP 1980; 7: 165 - 176

6. van Der Velden. Probing force and the relationship of the probe tip to the periodontal tissues. JCP 1979; 6: 106 $-114$

7. Abbas et al. Effect of training and probing force on the reproducibility of pocket depth measurements. $\mathbf{J}$ Periodontal Res 1982;17:226-234.

8. Miller H-P, Eger T: Furcation diagnosis. J Clin Periodontol 1999;26:485-498.

9. Moriarty JD,et al. Inter-examiner reproducibility of probing pocket depths in molar furcation sites. J Clin Periodontol 1988;15:68-72.

10. Eickholz P. Reproducibility and validity of furcation measurements as related to class of furcation invasion. $\mathrm{J}$ Periodontol 1995;66:984-989.

11. Eickholz P \& Stachele HJ. The reliability of furcation measurements. J Clin Periodontol 1994;21:611-614.

12. Reddy et al.A comparision of manual and controlled-forcet attachment-level measurements. J Clin Periodonlol 1997; $24:$ 920-926.

13. Grossi et al. Sources of error for periodonlal probing measurements. J Periodonl Res 1996; 31: $330-336$.

14. Breen et al. Important Differences in Clinical Data from Third, Second, and First Generation Periodontal Probes. JP 1997; 68: 335 - 345

15. Grbic et al (a). Risk indicators for future clinical attachment loss in adult periodontitis. Patient variables. JP 1991; 62: 322 -329

16. Grbic et al (b). Risk indicators for future clinical attachment loss in adult periodontitis. Tooth and site variables. JP 1992; 63: 262 -269 
17. Grossi et al. Assessment of risk for periodontal disease. I. Risk indicators for attachment loss. JP 1994; 65: 260 $-267$

18. Haffajee et al. Subgingival temperature (II). Relation to future attachment loss. JCP 1992; 19: 409 - 416

19. Machtei et al. Clinical criterion for the definition of "Established Periodontitis". JP 1992; 63: $207-215$

20. Hung et al. Meta-analysis of the effect of scaling and root planning, surgical treatment and antibiotic therapies on periodontal probing depth and attachment loss. JCP 2002; 29: 975 - 986

21. Alves et al. Clinical attachment loss produced by curettes and ultrasonic scalers. JCP 2005; 32: $691-694$

22. Orobitg et al. The relation between pocket depth change and attachment loss in subjects with moderate to severe periodontitis. J Dent Res 1996; 75: 211, abst 1549. 